\title{
MONOTHETIC ALGEBRAIC GROUPS
}

\author{
GIOVANNI FALCONE ${ }^{\complement}$, PETER PLAUMANN and KARL STRAMBACH
}

(Received 22 November 2004; revised 5 October 2005)

Communicated by E. A. O'Brien

\begin{abstract}
We call an algebraic group monothetic if it possesses a dense cyclic subgroup. For an arbitrary field $k$ we describe the structure of all, not necessarily affine, monothetic k-groups $G$ and determine in which cases $G$ has a k-rational generator.
\end{abstract}

2000 Mathematies subject classification: primary 14L99; secondary 20G15, $20 \mathrm{~K} 99$.

The abstract theory of groups, especially in the infinite case, deals preponderantly with the study of classes of groups which are given by distinguished group theoretical properties. For algebraic groups so far this approach has not been applied systematically. If one wants to follows the pattern given by the abstract theory of groups in the case of algebraic groups, it is indispensable to have detailed knowledge of those algebraic groups that correspond to cyclic groups.

Groups that have a dense cyclic subgroup play a fundamental role in the theory of topological groups. For the class of locally compact groups these groups are called monothetic and were introduced by van Dantzig in [14]. The full classification of their structure can be found in [4, Section 25].

In the theory of algebraic groups over a field $k$ we study the analogous class of groups, which we also call monothetic. So far, groups having a dense cyclic subgroup with respect to the Zariski topology have not found particular attention. In [10, page 146] the affine monothetic groups over an algebraically closed field of characteristic 0 are described in connection with the Galois groups of differential equations.

In order to treat algebraic groups with group theoretical methods it is necessary to know the monothetic groups in detail. We classify them for k-groups and find

Supported by D.F.G., M.I.U.R., Università di Palermo (Co.R.I.).

(C) 2007 Australian Mathematical Society 1446-7887/07 \$A2.00+0.00 
k-rational generators whenever they exist. Our results may be summarized by the following theorem.

MAIN THEOREM. (I) A k-group $G$ is monothetic if and only if it is the direct product of a connected monothetic $\mathrm{k}$-group $G^{\circ}$ and a finite cyclic group.

(II) A connected algebraic k-group $G \neq 1$ is monothetic if and only if $\mathrm{k}$ is not locally finite and $G=H D$, where $H$ is a monothetic connected affine k-group, $D$ is a connected monothetic algebraic k-group having no non-trivial affine image, and $H \cap D$ is finite.

(III) A connected algebraic k-group $G \neq 1$ having no non-trivial affine epimorphic image is monothetic if and only if $\mathrm{k}$ is not locally finite.

(IV) A connected affine algebraic k-group $H \neq 1$ is monothetic if and only if $\mathrm{k}$ is not locally finite and $H$ is the direct product of a torus $T$ and an $\epsilon$-dimensional connected unipotent group $V$, where $\epsilon=0$ if $\operatorname{char}(\mathrm{k})>0$, whereas $\epsilon \leq 1$ if $\operatorname{char}(\mathrm{k})=0$. Both groups $T$ and $V$ are defined over $\mathrm{k}$.

(V) A monothetic algebraic k-group $G$ has a k-rational generator if and only if the minimal subgroup $D$ of $G$ with an affine factor group has a k-rational generator.

In Theorem 16 we show that all closed connected subgroups of a monothetic algebraic group $G$ are monothetic if and only if the maximal connected unipotent subgroup $U$ of $G$ is monothetic. This condition is clearly satisfied for affine groups and for abelian varieties. The same holds over fields of positive characteristic (Corollary 15). However, in characteristic 0 , there are counter examples, which are connected monothetic non-affine groups having a vector subgroup of dimension greater than 1 (Remark 12).

Our paper shows that in contrast to the monothetic Lie groups, which are precisely the direct products of a torus and a cyclic discrete group, the structure of algebraic monothetic groups is more subtle.

For various discussions and hints we are grateful to Peter Müller from Würzburg. Furthermore we thank the referee to his truly helpful suggestions.

A. Prerequisites In this note we consider algebraic k-groups. A general reference for these groups is Borel's book [1]. If not specified, we denote by $G$ the set of elements which are rational over the algebraic closure $k^{a}$ of $k$. If we consider the rational points of $G$ over a field $F$ containing the minimal field of definition for $G$ (see [13] and [16, Chapter IV, Corollary 3, page 71]). we denote this set by $G(F)$.

We call an algebraic k-group $G$ monothetic if it contains an element $x$ such that $G=\overline{\langle x\rangle}$. In this case $G$ is a commutative group [1. Section 2.1(e)]. We call $x$ a generator of $G$.

Clearly any epimorphic image of a monothetic group is monothetic as well. Conversely we have the following result. 
PROPOSITION 1. Let $G$ be a connected algebraic k-group, and let $E$ be a finite subgroup of $G$ such that $G / E$ is monothetic. Then $G$ is monothetic also.

ProOF. Let $G / E=\overline{\langle u E\rangle}$ and $U=\overline{\langle u\rangle}$. Then $G=U E$. It follows $G=U^{\circ}$, the connected component of the identity in $U$. Thus $G$ coincides with $U$.

Proposition 1 shows that together with a connected algebraic group $G$ all groups isogenous to $G$ are monothetic.

Let $x$ be a generator of $G$. As the connected component $G^{\circ}$ of an algebraic group $G$ has finite index in $G$, a power $x^{m}$ of $x$ lies in $G^{\circ}$. Put $H=\overline{\left\langle x^{m}\right\rangle}$; then $H \leq G^{\circ}$. Since the finite union $H \cup H x \cup \ldots \cup H x^{m-1}$ is a closed subgroup containing $x$, it is all of $G$, and we have $H=G^{\circ}$. If $G$ is a k-group then $H=G^{\circ}$ is a k-group, too. Therefore, we arrive at the following result.

PROPOSITION 2. Let $G=\overline{\langle x\rangle}$ be a monothetic algebraic k-group. Then its connected component $G^{\circ}$ is a monothetic k-group also. If $G$ is connected, then for any integer $m$ we have $G=\overline{\left\langle x^{m}\right\rangle}$.

By this proposition we are justified to restrict our attention mostly to connected monothetic k-groups. We shall discuss the non-connected case in more detail later.

The connected subgroups of a connected monothetic Lie group are always monothetic. In the case of connected algebraic $k$-groups this is true for affine groups (Corollary 6), as well as for abelian varieties (Lemma 9), but not in general (Remark 12). Of course, subgroups that are not connected do not have to be monothetic.

The set $G(\mathrm{k})$ of k-rational elements of an algebraic k-group $G$ is always a subgroup of $G=G\left(\mathrm{k}^{a}\right)$ ([15, Sections I.7 and II.8-10]). We are interested in the question of whether it is possible to find a k-rational generator of a given monothetic k-group $G$. It follows from the next proposition that this question is meaningful only if the field $k$ is not locally finite.

\section{PROPOSITION 3. Let $G$ be a k-group.}

(a) If $\mathrm{k}$ is a locally finite field, then $G(\mathrm{k})$ is a torsion group.

(b) If $G$ has positive dimension and is monothetic, then the field $\mathrm{k}$ is not locally finite and there is a finite extension $\mathrm{F}$ of $\mathrm{k}$ such that $G\left(\mathrm{k}^{a}\right)$ contains an $\mathrm{F}$-rational generator.

PROOF. Observe that the minimal field $k_{0}$ of definition for $G$ is finitely generated over the prime field of $k$, since the representatives of $G$ are described by finitely many polynomials (see [16, page 179]). Consider $a \in G\left(k^{a}\right)$. Adjoining to $k_{0}$ the set of coordinates of $a$ with respect to a finite system of charts for $G$, one obtains a finite extension $\mathrm{k}_{0}(a)$ of $\mathrm{k}_{0}$, over which $a$ is rational and $G$ is defined (compare [16, Chaper VII.3]). 
(a) If $\mathrm{k}$ is locally finite, then for $g \in G(\mathrm{k})$ the field $\mathrm{k}_{0}(g)$ is finite. Thus $g$ lies in the finite group $G\left(\mathrm{k}_{0}(g)\right)$.

(b) Obviously any generator $g$ of a k-group $G$ of positive dimension has infinite order. Because of (a), the field $k$ is not locally finite. Since $k_{0}$ is a subfield of $k$, the field $F=k(g)$ has finite degree over $k$.

B. Affine groups For affine groups the structure theorems for algebraic k-groups, found in [1, Chapter III] enable one to determine the structure of monothetic groups and to consider rationality questions.

PROPOSITION 4. A connected unipotent $\mathrm{k}$-group $G \neq 1$ is monothetic if and only if $\operatorname{char}(\mathrm{k})=0$ and $\operatorname{dim}(G)=1$. In this case every element of $G$ that is not equal to 1 is a generator of $G$, and $G$ has k-rational generators.

PROOF. If $\operatorname{char}(\mathrm{k})>0$, then $G$ is a torsion group. If $\operatorname{char}(\mathrm{k})=0$, then every element of $G$ that is not equal to 1 has infinite order and generates a 1-dimensional subgroup of $G$.

THEOREM 5. A connected affine algebraic k-group $G \neq 1$ is monothetic if and only if the following conditions hold:

(a) the field $\mathrm{k}$ is not locally finite;

(b) $G$ is commutative; and

(c) the unipotent radical $G_{u}$ is an $\epsilon$-dimensional vector group, where $\epsilon=0$ if $\operatorname{char}(\mathrm{k})>0$ and $\epsilon \leq 1$ if $\operatorname{char}(\mathrm{k})=0$.

In this case, the unipotent radical $G_{u}$ and the maximal torus $G_{s}$ of $G$ are defined over $\mathrm{k}$ and $a$ generator of $G$ can be chosen to be k-rational.

ProOF. Let $G$ be a monothetic k-group. Since $G$ is commutative, it is the direct product of $G_{u}$ and $G_{s}$, where $G_{u}$ is the set of unipotent elements of $G$, which is a k-closed subgroup, and $G_{s}$ is the set of its semisimple elements, which is even defined over $\mathrm{k}$ (see [1, Theorems 4.7 and 10.6 (3)]). For a generator $x$ of $G$ one has $x=x_{u} x_{s}$ with $x_{u} \in G_{u}$ and $x_{s} \in G_{s}$. Hence $G=\overline{\left\langle x_{u}\right\rangle} \times \overline{\left\langle x_{s}\right\rangle}, G_{s}=\overline{\left\langle x_{s}\right\rangle}$ and $G_{u}=\overline{\left\langle x_{u}\right\rangle}$.

It follows from Proposition 4 that (c) holds. Since fields of characteristic 0 are perfect, the unipotent radical of a monothetic affine k-group is defined over $k$ (see [5, Lemma 34.1, page 217]) and we can choose a generator of $G_{u}$ that is rational over k (see Proposition 4).

The subgroup $G_{s}$ of the monothetic k-group $G$ is a k-torus. From [1, Proposition 8.8 and Remark] it follows that a k-torus is monothetic if and only if $k$ is not locally finite. In this case, a generator can be chosen to be k-rational.

Conversely, let $G$ be an affine k-group satisfying (a)-(c). Then $G=G_{u} \times G_{s}$ and $G_{u}, G_{s}$ are k-groups with k-rational generators $x_{u}, x_{s}$. Consider the monothetic group 
$X=\overline{\left\langle x_{u} x_{s}\right\rangle}$. Then the restrictions to $X$ of the projections from $G$ to $G_{u}$ and from $G$ to $G_{s}$, respectively are surjective. Since there are no non-trivial homomorphisms between $G_{u}$ and $G_{s}$ it follows that $G=X$ (compare [9; page 40]).

COROLLARY 6. Every closed connected subgroup of an affine connected monothetic algebraic k-group is monothetic.

C. Abelian varieties We now turn our attention to monothetic abelian varietes. A simple abelian variety is monothetic if and only if it is not a torsion group. The next statement, which is a variant of Theorem 12 in [2], is needed for the proof of our Theorem 9, where we show that also for abelian varieties the converse of Proposition 3 holds.

A field $k$ is called Hilbertian if Hilbert's irreducibility theorem holds over $k$ (compare [3, page 141]). Important examples of Hilbertian fields are finitely generated transcendental extensions of some subfield (compare [3, Theorem 12.10, page 155]) and the algebraic number fields (compare [3, Corollary 12.8, page 154]).

THEOREM 7. Let $\mathrm{k}$ be a field that is not locally finite and let $A \neq 1$ be an abelian variety, defined over $\mathrm{k}$. Then there is a Hilbert field $\mathrm{E} \subseteq \mathrm{k}$ such that $A$ is defined over $\mathrm{E}$ and $A\left(\mathrm{E}^{a}\right)$ has infinite torsion free rank. The field $\mathrm{E}$ is finitely generated over its prime field.

PROOF. Assume that the torsion free rank of $A\left(\mathrm{k}^{a}\right)$ is some finite number $d \geq 0$. Let $L$ be an algebraically closed extension of $k$ of uncountable transcendence degree. Since the torsion subgroup of $A(\mathrm{~L})$ is countable (see [8, page 39]), whilst $A(\mathrm{~L})$ itself has the same cardinality as $\mathrm{L}$, there are elements $a_{1}, \ldots, a_{d+1}$ in $A(\mathrm{~L})$, that generate a free abelian group of rank $d+1$. If we embed $A(L)$ into a projective space over $\mathrm{L}$, we see that there exists a field $F$, finitely generated over the prime field of $k$, such that the elements $a_{1}, \ldots, a_{d+1}$ are rational in $A(F)$.

Let $\mathrm{k}_{0}$ be a minimal field of definition for $A$ contained in $\mathrm{k}$. Since $A$ is defined (in any of its finitely many charts) by polynomials with coefficients in $k_{0}$, the field $k_{0}$ is finitely generated. We assume first that $k_{0}$ is locally finite. Then there exists an element $t_{0}$ in $\mathrm{k}$, which is transcendental over $\mathrm{k}_{0}$. We put $\mathrm{E}=\mathrm{k}_{0}\left(t_{0}\right)$. If $\mathrm{k}_{0}$ is not locally finite, we put $E=k_{0}$. In both cases the field $E$ is Hilbertian, contained in $k$, and is a field of definition for the abelian variety $A$.

By a theorem of Néron [7, Chapter 1, Theorem 7.2, page 41], there exists an injective specialization homomorphism $\sigma$ from $A(F)$ to $A\left(E^{a}\right)$. Hence $\left\{\sigma\left(a_{1}\right), \ldots, \sigma\left(a_{d+1}\right)\right\}$ are $\mathbb{Z}$-independent elements in $A\left(\mathrm{E}^{a}\right) \subset A\left(\mathrm{k}^{a}\right)$ : a contradiction.

Proposition 8. Assume that the field $\mathrm{k}$ is not locally finite and let $A_{1}, A_{2}$ be abelian k-varieties. Then for every element $a_{1} \in A_{1}$ of infinite order there exists an element 
$a_{2} \in A_{2}$ of infinite order such that $\varphi\left(a_{1}\right) \neq a_{2}{ }^{n}$ for all algebraic homomorphisms $\varphi$ from $A_{1}$ to $A_{2}$ and for any $n \in \mathbb{N}$.

PROOF. By [8, Theorem 3, page 176], the group $\operatorname{Hom}\left(A_{1}, A_{2}\right)$ of all algebraic homomorphisms from $A_{1}$ to $A_{2}$ has finite torsion free rank. Thus the abstract group $X=\operatorname{Hom}\left(A_{1}, A_{2}\right) a_{1}$ is a commutative group of finite rank. It follows from Theorem 7 that the group $X$ cannot intersect all torsion-free subgroups of rank 1 of $A_{2}$ nontrivially.

Now we treat monothetic abelian varieties in general. Although the discussion of monothetic abelian varieties is in the spirit of Borel's proof for tori (see [1, Proposition 8.8]), it becomes more complicated.

THEOREM 9. An abelian $\mathrm{k}$-variety $A \neq 1$ is monothetic if and only if the field $\mathrm{k}$ is not locally finite.

PROOF. If $\mathrm{k}$ is locally finite, then $A$ is a torsion group by Proposition 3 . Conversely, assume that $\mathrm{k}$ is not locally finite and that $A$ is a counterexample of minimal dimension. By Theorem 7, the abelian variety $A$ is not simple, hence $A=A_{1} A_{2}$, where $A_{1}$ is a simple non-trivial abelian variety and $A_{2}$ is monothetic, by minimality. Because of Proposition 1 we may assume that $A=A_{1} \times A_{2}$. Choose an arbitrary generator $t_{2}$ of $A_{2}$ and an element $t_{1} \in A_{1}$ of infinite order such that $t_{1}{ }^{n} \neq \varphi\left(t_{2}\right)$ for any $n \in \mathbb{N}$ and for all algebraic homomorphisms $\varphi$ from $A_{2}$ to $A_{1}$. This is possible thanks to Proposition 8 . We put $U=\overline{\left\langle\left(t_{1}, t_{2}\right)\right\rangle}$. Then $U \neq A$ and, changing $\left(t_{1}, t_{2}\right)$ to a suitable power, by Proposition 2 we can even assume that $U$ is connected. Since $A_{1}$ is simple, the group $U \cap A_{1}$ has some finite order $m$.

Let $\pi_{i}: U \rightarrow A_{i}$ be the restriction to $U$ of the canonical projection from $A$ to $A_{i}$, $(i=1,2)$. Since $t_{2}=\pi_{2}\left(t_{1}, t_{2}\right) \in A_{2}$, the homomorphism $\pi_{2}$ is surjective; its kernel is the finite group $U \cap A_{1}$ of order $m$. It follows from [8, Chapter IV.18, Remark, page 169] that there is a homomorphism $\eta: A_{2} \rightarrow U$ such that $\eta \pi_{2}\left(x_{1}, x_{2}\right)=\left(x_{1}, x_{2}\right)^{m}$ for all $\left(x_{1}, x_{2}\right) \in U$. But then

$$
\pi_{1} \eta\left(t_{2}\right)=\pi_{1} \eta \pi_{2}\left(t_{1}, t_{2}\right)=\pi_{1}\left(t_{1}, t_{2}\right)^{m \prime}=\left(\pi_{1}\left(t_{1}, t_{2}\right)\right)^{m}=t_{1}^{m},
$$

a contradiction to the choice of $t_{1}$.

In contrast to the affine case (Theorem 5 ), in general we cannot say anything more about the rationality of a generator of an abelian k-variety $A$ than we said in Proposition 3. For $k=\mathbb{Q}$ one finds in [6, Chapter I.3, Table 2] examples of elliptic curves, where the group of $\mathbb{Q}$-rational points is finite. Using [6, Theorem 3.3, page 34] one sees that are many cases in which the identity is the only $\mathbb{Q}$-rational point of $A$. One such example is given by the equation $y^{2}=x^{3}+6$. 
D. The general case In [11], Rosenlicht described how an arbitrary connected algebraic group is built from an affine group and an abelian variety. We collect these facts in the following theorem.

THEOREM 10 (Rosenlicht [11]). Let $G$ be a connected algebraic k-group. Then there exists a (unique) maximal connected affine subgroup $L_{G}$ and $a$ (unique) minimal (connected) normal subgroup $D_{G}$ such that the factor group $G / D_{G}$ is affine. The following statements hold:

(i) $G=L_{G} D_{G}$.

(ii) $L_{G}$ is a k-closed characteristic subgroup of $G$, and $G / L_{G}$ is an abelian variety.

(iii) $D_{G}$ is defined over $\mathrm{k}$ and is central and characteristic in $G$. It has no nontrivial affine epimorphic image, and it has only a finite number of elements of any given finite order.

(iv) Any k-closed abelian subvariety $A$ of $G$, as well as the connected component of $L_{G} \cap D_{G}$, is defined over $\mathrm{k}$ and is contained in $D_{G}$. Moreover, for the subgroup $A$ there exists a connected k-closed algebraic subgroup $G_{1}$ of $G$ such that $G=G_{1} A$ and $G_{1} \cap A$ is finite.

By the preceding theorem the group $D_{G}$ is commutative. Hence the connected component of $L_{G} \cap D_{G}$ is the direct product of a vector group and a torus ([5, Theorem 15.5, page 100]).

LEMMA 11. If the field $\mathbf{k}$ is not locally finite, then a connected algebraic $\mathrm{k}$-group $G$ having no non-trivial affine epimorphic image is always monothetic. If $G$ is such a group and $\mathrm{k}$ has positive characteristic, then $L_{G}$ is a torus.

PROOF. Let $L$ be the maximal connected affine subgroup of $G$. As $G / L$ is an abelian variety, by Theorem 9 , we find an element $y \in G / L$ such that $G / L=\overline{\langle y\rangle}$. Let $x$ be a pre-image of $y$ with respect to the canonical projection $G \rightarrow G / L$. We put $X=\overline{\langle x\rangle}$. Since $X L / L$ contains $y$, we see that $X L=G$. Thus $G / X=X L / X \cong L /(L \cap X)$ is affine and consequently we have $G=X$.

Assume finally that char(k) is positive. Since $G$ has only a finite number of elements of order $p$ (Theorem 10 (iii)), the group $G$ in this case cannot contain any non trivial vector subgroup.

If a monothetic k-group $G$ having no non-trivial affine epimorphic image is an extension of a vector group $H$ by an abelian variety $A$, then it follows from Lemma 11 that the field $\mathrm{k}$ has characteristic 0 . Conversely, if $\operatorname{char}(\mathrm{k})=0$, then according to [12, Proposition 11 and Theorem 3] such extensions exist for $\operatorname{dim}(H) \leq \operatorname{dim}(A)$. From our Theorem 5 we see that the vector group $H$ is monothetic if and only if $\operatorname{dim}(H) \leq 1$. Hence, in contrast to Corollary 6 , we have the following observation. 
REMARK 12. Closed connected subgroups of connected monothetic k-groups $G$ are not necessarily monothetic if the maximal connected affine subgroup of $G$ is not a torus. This can only happen if $\operatorname{char}(k)=0$.

THEOREM 13. A connected algebraic k-group $G \neq 1$ is monothetic if and only if the field $\mathrm{k}$ is not locally finite and $G$ is a product $D H$ with finite $D \cap H$, where $H$ is a monothetic connected affine $\mathrm{k}-$ group and $D$ is a monothetic connected $\mathrm{k}$-group having no non-trivial affine epimorphic image.

A connected algebraic k-group $G$ has a k-rational generator if and only if $D$ has a k-rational generator; the group $H$ always has a k-rational generator.

PROOF. Assume first that $G$ is monothetic, and consider the subgroups $L=$ $L_{G}, D=D_{G}$ of $G$ as in Theorem 10. There is a finite subgroup $E$ of $G$ such that $G / E=C \times D_{G / E}$ with an affine group $C$ ([9, Lemma 3]). Thus by Proposition 1, we may suppose that $L \cap D$ is connected. Furthermore, $L$ is a k-closed subgroup of $G$ by Theorem 10 (ii) and there is a complement $H$ of $L \cap D$ in $L$; in particular, we have $H \cap D=1$. The affine algebraic group $H$ is isomorphic to $L /(L \cap D)$.

If $\operatorname{char}(\mathrm{k})=0$, then the affine monothetic group $G / D=L D / D$ is the direct product of a torus with an $\epsilon$-dimensional vector group $(\epsilon \leq 1)$ by theorem 5 . By Theorem 10 (ii) and [5, Lemma 34.1, page 217], the group $L$ is a connected commutative affine algebraic k-subgroup of $G$.

If char $(\mathrm{k})>0$, then $L D / D$ is a torus (Theorem 5) and $D$ is an extension of a torus by an abelian variety (Lemma 11 ); in particular, $L$ is the maximal torus of $G$. By [11, Proposition 4, page 443], it follows that $L$ is defined over k.

Now, in both cases, the group $H \cong L /(L \cap D)$ is defined over $\mathrm{k}$ ([11, Theorem 4 , page 413]) and is monothetic as an epimorphic image of $G$. Finally, we observe that $G$ is isogeneous to the direct product of $H$ and $D$.

Conversely, let $G=H D$ with k-groups $D=\overline{\langle x\rangle}, H=\overline{\langle y\rangle}$. Since $H \cap D$ is finite, we may assume that $H \cap D=1$. Consider the group $U=\overline{\langle x y\rangle}$. If $U \neq G$, then $D$ and $H$ are not contained in $U$.

Observing that $G=D \times H$ we consider the subgroup $N=(D \cap U) \times(H \cap U)$ and the factor group

$$
G_{1}=G / N=(D \times H) /((D \cap U) \times(H \cap U)) \cong D_{1} \times H_{1},
$$

where $D_{1}=D /(D \cap U)$ is the minimal subgroup of $G_{1}$ with an affine factor group and $H_{1}=H /(H \cap U)$ is the maximal connected affine subgroup of $G_{1}$. With $U_{1}=U / N$, we have $U_{1} \times D_{1}=G_{1}=U_{1} \times H_{1}$. Since $G_{1} / D_{1}$ is affine, it follows that $U_{1}$ is affine. Hence $G_{1}=U_{1} H_{1}$ is affine, a contradiction. Thus $D$ or $H$ is contained in $U$, but in both cases $G=U$ is monothetic. 
Since the monothetic group $H$ is defined over k, it follows from Theorem 5 that we can choose a k-rational generator $x$ for $H$. If $y$ is a k-rational generator of $D$, then, as just shown, the element $x y$ is a k-rational generator of $G$. Conversely, if $z$ is a k-rational generator for $G$, then for any k-rational generator $x$ for $H$ the element $x^{-1} z$ is a k-rational generator of $D$.

COROLlaRY 14. A connected monothetic k-group $G$ is divisible (as an abstract commutative group).

ProOF. We consider monothetic subgroups $D, H$ of $G$ satisfying $G=D H$ as in Theorem 13. It follows from Lemma 11 that $D$ is divisible as an extension of a divisible group by a divisible group. Since $H$ is divisible by Theorem 5 , the assertion follows.

From the preceding theorem, Corollary 6 and Remark 12, we also obtain the following result.

COROLLARY 15. Let $\mathrm{k}$ be a field. Each closed connected subgroup of every monothetic $\mathrm{k}$-group is monothetic if and only if $\mathrm{k}$ has positive characteristic.

For fields of characteristic 0 , our structure theorems, together with Remark 12 yield the following.

COROLLARY 16. All closed connected subgroups of a monothetic k-group $G$ over a field of characteristic 0 are monothetic if and only if the maximal connected unipotent subgroup of $G$ has dimension at most 1.

THEOREM 17. An algebraick-group $G$ is monothetic if and only if $G$ is commutative, the connected component $G^{\circ}$ is monothetic, and $G / G^{\circ}$ is a finite cyclic group.

PROOF. If $G$ is monothetic, then $G^{\circ}$ is monothetic by Proposition 2 and $G / G^{\circ}$ is a finite cyclic group.

Conversely, let $G^{\circ}$ and $G / G^{\circ}$ be monothetic. Since $G^{\circ}$ is divisible by Corollary 14 , there is a finite cyclic subgroup $F \cong G / G^{\circ}$ of $G$ such that $G=G^{\circ} \times F$ is an algebraic group. Let $g$ be a generator of $G^{\circ}$ and let $f$ be a generator of $F$. Using Proposition 2 it follows that $g f$ is a generator of $G$.

REMARK 18. Let $G=G^{\circ} \times F$ be a non-connected monothetic k-group. One has the decomposition $G^{\circ}=H D$ as in Theorem 13 , since $G^{\circ}$ is a monothetic k-group by Proposition 2. The group $H \times F$ is a monothetic affine k-group (see [11, Corollary 1 , page 430]). Using Theorem 5, one sees that $H \times F$ has a k-rational generator. Thus $G$ has a k-rational generator if and only if $D$ has this property. 


\section{References}

[1] A. Borel, Linear algebraic groups, Graduate Texts in Mathematics 126 (Springer-Verlag, New York, 1991).

[2] G. Frey and M. Jarden, 'Approximation theory and the rank of abelian varieties over large algebraic fields', Proc. Lond. Math. Soc. (3) 28 (1974), 112-128.

[3] M. Fried and M. Jarden, Field Arithmetic (Springer-Verlag, Berlin, 1986).

[4] E. Hewitt and K. A. Ross, Abstract harmonic analysis. Vol. I: Structure of topological groups. Integration theory. Group representations (Springer-Verlag, Berlin, 1963).

[5] J. E. Humphreys, Linear algebraic groups, Graduate Texts in Mathematics 21 (Springer-Verlag, New York, 1975, 1981).

[6] D. Husemöller, Elliptic curves, Graduate Texts in Mathematics 111 (Springer-Verlag, New York, 1987).

[7] S. Lang, 'Number theory III: Diophantine geometry', Encyclopaedia of Mathematical Sciences 60 (ed. R. V. Gamkrelize) (Springer-Verlag, Berlin, 1991).

[8] D. Mumford, Abelian varieties (Oxford University Press, Oxford, 1974).

[9] P. Plaumann, K. Strambach and G. Zacher, 'Der Verband der zusammenhängenden Untergruppen einer kommutativen algebraischen Gruppe', Arch. Math. (Basel) 85 (2005), 37-48.

[10] M. van der Put and M. F. Singer, Galois theory of linear differential equations, Grundlehren der Mathematischen Wissenschaften 328 (Springer-Verlag, Berlin, 2003).

[11] M. Rosenlicht, 'Some basic theorems on algebraic groups', Amer. J. Math. 78 (1956), 401-443.

[12] — 'Extensions of vector groups by abelian vareties', Amer. J. Math. 80 (1958), 685-714.

[13] - 'The definition of field of definition', Bol. Soc. Mat. Mexicana (2) 7 (1962), 39-46.

[14] D. van Dantzig, 'Über topologisch homogene Kontinua', Fund. Math. 15 (1930), 102-125.

[15] A. Weil, Variétés abéliennes et courbes algébriques (Hermann \& Cie, Paris, 1948).

[16] - Foundations of algebraic geometry (American Mathematical Society, Providence, RI, 1962).

Dipartimento di Metodi e Modelli Matematici

Università di Palermo

viale delle Scienze

I-90128 Palermo

Italy

e-mail: gfalcone@unipa.it
Mathematisches Institut Universität Erlangen Bismarckstraße 1 1/2 D-91054 Erlangen Germany e-mail: plaumann@mi.uni-erlangen.de strambach@mi.uni-erlangen.de 